\title{
Management of insomnia in primary care
}

\section{Lauren $\mathbf{N g}$ (iD \\ Sleep medicine fellow \\ David Cunnington (D) \\ Sleep physician? \\ 1 Melbourne Sleep Disorders Centre}

\section{Keywords}

benzodiazepines, cognitive behavioural therapy, hypnotics, insomnia, sleep disturbance, sleep-wake disorders

Aust Prescr 2021;44:124-8 https://doi.org/10.18773/ austprescr.2021.027

\section{Corrected}

Tuesday 10 August 2021

This is the corrected version of the article.

Correction notice available at:

https://doi.org/10.18773/ austprescr.2021.043

\section{SUMMARY}

Insomnia can have significant health and economic impacts. In contrast, sleep disturbance is common but does not usually affect daytime activity.

Short-term approaches for acute insomnia are often appropriate. These include dealing with precipitating factors such as stress.

Chronic insomnia has a high relapse and recurrence rate. It is best managed with cognitive behavioural therapy which includes sleep hygiene, stimulus control and sleep restriction.

In primary care, brief behavioural therapy for insomnia is an accessible and effective management strategy. If there is no response, referral should be considered.

Adjuvant use of drugs in insomnia may be appropriate in some cases. Prescription should be for a limited duration.

\section{Introduction}

Insomnia is a common sleep disorder involving difficulty initiating or maintaining sleep and results in daytime consequences (see Box). ${ }^{1}$ Chronic insomnia is the most prevalent sleep disorder and affects $12.2 \%$ of Australian adults. It is often under-recognised with only $7.5 \%$ reporting that they have had a diagnosis by a medical professional. ${ }^{2}$ Inadequate sleep and chronic insomnia are associated with a high burden

\section{Box Diagnostic criteria of insomnia'}

Dissatisfaction with sleep quality or quantity associated with one or more of the following:

- difficulty initiating sleep

- difficulty maintaining sleep (frequent awakenings or problems returning to sleep after awakening)

- early morning awakening with inability to return to sleep.

Disturbance causes clinically significant distress or impairment (social, occupational, educational, academic, behavioural or other important areas of functioning).

Frequency of at least three nights per week.

Duration more than three months.

Occurs despite adequate opportunity for sleep.

Is not better explained by, and does not occur exclusively during, the course of another sleep-wake disorder (e.g. narcolepsy, breathing-related sleep disorder, a circadian rhythm sleep-wake disorder, parasomnia).

Coexisting mental disorders and medical conditions do not adequately explain the predominant complaint of insomnia.

Insomnia is not attributable to the physiological effects of a substance (e.g. drug of abuse or medicine). of disease with an increased risk of depression, cardiovascular disease and death. ${ }^{3-5}$ People suffering from insomnia have greater work absenteeism, reduced productivity and are more likely to access healthcare with increased presentations to general practice and hospital. 6,7

The cost of insufficient sleep in Australia is significant. The associated healthcare costs are $\$ 1.24$ billion with an estimated $\$ 12.19$ billion in lost productivity. ${ }^{8}$

\section{Evaluation and diagnosis}

It is important to take a thorough history to distinguish between insomnia and sleep disturbance. While sleep disturbance, with difficulty initiating and maintaining sleep, is a common complaint, many people continue to function well throughout the day. In contrast, insomnia has an impact on daytime functioning. 'Presentations with sleep disturbance stem from a belief that sleep is failing to meet expectations rather than having any true negative consequences. This belief is often perpetuated by society's emphasis on the importance of obtaining perfect sleep for optimal health and well-being.

\section{Acute insomnia}

Short-term insomnia with sleep disturbance and daytime impacts for less than three months will often occur in response to a precipitating stressor which results in a predictable change in sleep quality. Acute insomnia is at risk of evolving into chronic insomnia if individuals develop changed thinking and maladaptive behaviours around sleep. ${ }^{9}$ For example, if people are not sleeping well, it is common for them to retire to bed earlier wishing to get to sleep, which leads to spending longer in bed getting frustrated about not 
sleeping. People can also start using countermeasures like caffeine to reduce tiredness during the day, which can negatively impact on sleep on subsequent nights.

\section{Chronic insomnia}

Chronic insomnia or insomnia disorder is defined as a sleep disturbance occurring on three or more nights per week, over a three-month period, which results in significant distress or impacts on daytime functioning.

There is a high rate of recurrence so a previous history of insomnia is a significant predictor of the future development of insomnia disorder. ${ }^{10}$ In a longitudinal study of 388 patients with chronic insomnia, $46 \%$ of patients had persistent symptoms at three years and $14 \%$ had a relapse following remission of their insomnia."

\section{Comorbidities}

Comorbid disorders such as depression, anxiety and chronic medical conditions are common. ${ }^{12}$ As insomnia and comorbidities can impact on each other, comprehensive assessment and management of all contributing conditions is essential.

Insomnia may also present in conjunction with a comorbid sleep disorder. Up to 50\% of people with obstructive sleep apnoea will report symptoms of insomnia and may have difficulty in adhering to continuous positive airway pressure (CPAP) therapy. ${ }^{13}$ While tiredness and fatigue are common symptoms of insomnia, excessive daytime sleepiness is uncommon and warrants further investigation and exclusion of sleep disordered breathing. Other sleep disorders such as restless legs syndrome and circadian rhythm disorders can also present as insomnia.

\section{Initial management}

In patients with acute insomnia, a short-term approach to improve sleep quality is appropriate. This may involve strategies to reduce acute distress and possibly a short course of a hypnotic drug. Given the poorer prognosis with chronic insomnia, longer term management strategies are required to improve outcomes.

The key principles in the management of insomnia include reducing sleep-related anxiety and maladaptive behaviours around sleep, in addition to addressing comorbidities and precipitating factors. Education about sleep is an important component, addressing unrealistic or misinformed expectations about sleep which often perpetuate symptoms of insomnia. A stepped-care approach to insomnia in primary care may be appropriate given the high prevalence of insomnia and limited numbers of specialist sleep medicine and sleep psychology practitioners. ${ }^{14,15}$

\section{Cognitive behavioural therapy}

Cognitive behavioural therapy for insomnia is recommended as first-line therapy. It has five components (see Table): ${ }^{16}$

- sleep hygiene

- sleep restriction

- stimulus control

- relaxation strategies

- cognitive therapy.

Sleep hygiene aims to promote healthy behaviours and a conducive environment to improve sleep quality. However, there is limited evidence supporting sleep hygiene as a sole behavioural strategy and patients are at risk of developing maladaptive behaviours around sleep. ${ }^{17}$

Sleep restriction is a behavioural strategy that aims to reduce the time spent awake in bed by matching total time in bed with estimated sleep duration. A randomised trial involving 45 patients with chronic insomnia reported an improvement in symptoms in $73 \%$ of those receiving sleep restriction therapy and sleep hygiene compared with $35 \%$ of a control group receiving sleep hygiene alone. ${ }^{18}$

Stimulus control strategies aim to improve sleep quality by implementing a consistent sleep-wake schedule and strengthening cues that promote sleep. Strategies include directing patients to only go to bed when sleepy and leaving the bedroom to engage in another activity if they fail to fall asleep or wake during the night. These strategies attempt to reduce sleep-related anxiety and a conditioned response around sleep.

Brief behavioural therapy for insomnia combines sleep restriction and stimulus control strategies. In a randomised controlled trial of 82 patients there was remission in $36 \%$ and a response in $60 \%$ of patients after six months of follow-up..$^{19}$ The therapy consists of two face-to-face sessions, with an assessment and intervention session for 45-60 minutes and a 30-minute follow-up session after two weeks. As it does not require specialist training and can be delivered in an efficient manner, the Royal Australian College of General Practitioners supports its use by GPs and practice nurses. ${ }^{20}$

In patients who do not respond to brief behavioural therapy, cognitive therapies for insomnia may be required. Referral to a sleep psychologist should be considered.

Cognitive behavioural therapy is an effective long-term intervention, with a meta-analysis reporting significant improvement in sleep onset latency and sleep efficiency extending to 12 months. ${ }^{21}$ It is generally delivered weekly for two to eight sessions by a psychologist or trained health professional. However, there can be a significant barrier to access in primary care given the shortage of specialist sleep medicine and sleep psychology practitioners. 


\section{Table Components of cognitive behavioural therapy for insomnia}

\begin{tabular}{ll} 
Components & Rationale \\
\hline Sleep hygiene & $\begin{array}{l}\text { Promotes healthy bedtime } \\
\text { behaviours and an ideal } \\
\text { environment for sleep }\end{array}$ \\
& \\
Sleep/time in bed restriction & \\
& $\begin{array}{l}\text { Aims to balance estimated total } \\
\text { sleep time with opportunity to } \\
\text { sleep. This reduces the unnecessary } \\
\text { amount of time spent in bed awake } \\
\text { and increases sleep drive and } \\
\text { sleep debt to consolidate sleep on } \\
\text { subsequent nights }\end{array}$ \\
& \\
& \\
&
\end{tabular}

Stimulus control

Promotes a consistent sleep-wake schedule and reduces conditioned response around sleep

\section{Directions}

Behaviours:

- Avoid caffeine and nicotine before bedtime

- Maintain a regular bed and wake time

- Take regular daytime exercise

Environment:

- Maintain bedroom as a place to sleep

- Reduce noise and light

- Control temperature

- Avoid bedroom clutter

Assess patient's natural time of feeling sleepy and estimated total sleep time overnight (a sleep diary is often helpful in this setting).

Schedule a fixed bed and wake time based on estimated total sleep time, with at least six hours of opportunity for sleep, aiming for no longer than 30 minutes of wakefulness.

Maintain a consistent sleep-wake schedule regardless of how much sleep is obtained overnight

Lengthen sleep window in 30-minute increments on subsequent reviews depending on progress

Avoid naps during the day to increase natural homeostatic sleep drive Only go to bed when sleepy

If unable to fall asleep or waking for prolonged periods during the night, engage in another activity

Only return to sleeping position or bed when sleepy

Meditation

Breathing exercises

Progressive muscle relaxation

Guided visualisation practices

Implement short relaxation periods at various times during the day

Cognitive therapy

Encourages thought restructuring around sleep and promotes mindfulness
Challenge unhelpful beliefs and attitudes around sleep including the requirement for a certain number of hours of sleep, the health impacts of insomnia and attributional biases attached to inadequate sleep

\section{Drug management}

Up to $40 \%$ of patients with insomnia will have ongoing symptoms despite cognitive behavioural therapy. ${ }^{22,23}$ If drug therapy is considered, it is important to evaluate the underlying nature of the patient's insomnia and what the intended outcome of prescribing is.

\section{Acute insomnia}

Short-term drug therapy may be appropriate in acute insomnia with an identifiable precipitating stressor, illness or circumstances, in order to prevent progression to chronic insomnia. This would generally be of short duration, up to four weeks, while in parallel working on addressing the acute precipitating circumstances.

\section{Chronic insomnia}

In patients with chronic insomnia and high levels of distress, using a drug as an adjunct to cognitive behavioural therapy can be helpful to provide predictability with sleep and prevent escalation of sleep-related anxiety. In a trial of 160 adults with chronic insomnia, patients were randomised to receive cognitive behavioural therapy alone, or in conjunction with zolpidem for the first six weeks. The group that had combined therapy for six weeks, then cognitive behavioural therapy alone during six months of extended therapy, had the best outcomes with remission rates of $68 \%$ at six months. ${ }^{22}$ This study demonstrated the importance of addressing distress associated with insomnia early in treatment 
(first six weeks in this study), while people are working through cognitive behavioural therapy and increasing their skills and confidence in the cognitive and behavioural strategies. However, when prescribing any medicine in chronic insomnia it should be discussed with patients that the medicine is only intended as being short term as effects generally reduce over time.

Melatonin (2 $\mathrm{mg}$ extended-release formulation) is effective in treating insomnia in adults over the age of 55 over a three-week period. ${ }^{24}$ It may also be useful in patients with a circadian rhythm disturbance, resulting in sleep onset insomnia or early morning awakening. ${ }^{24,25}$ However, there is little evidence to guide formulation, dose and duration of therapy when used outside of melatonin's registered indication of insomnia in adults over the age of 55 years.

Suvorexant, a dual orexin receptor antagonist, can be an effective treatment in sleep maintenance insomnia due to hyperarousal. It can improve sleep latency, sleep maintenance and total sleep time compared to placebo. ${ }^{23,26}$

Benzodiazepines and benzodiazepine receptor agonists, including zolpidem and zopiclone, are effective short-term therapies for sleep onset and sleep maintenance insomnia. Data on long-term efficacy are limited. . $^{23,27}$

There is a risk of drug dependency, tolerance and abuse, but limiting dose escalation and regular monitoring are likely to mitigate this risk. ${ }^{23}$ When prescribing these drugs for insomnia, a harm-benefit assessment should be undertaken, with particular care in the elderly population. Sleep-promoting medicines can interact with other drugs that cause sedation, including alcohol, and should be used cautiously in these circumstances. Hypnotic drugs may increase the risk of falls, but so does a lack of sleep.

Despite a lack of evidence, antidepressants, sedating antipsychotics and antihistamines are often prescribed for the treatment of insomnia despite having significant potential adverse effects..$^{28-30}$ These drugs are not recommended because of this limited evidence and their adverse effects. ${ }^{23}$

\section{Stopping drugs}

Benzodiazepines and benzodiazepine receptor agonists are only recommended for short-term use, up to four weeks, so a strategy for their safe withdrawal is required. In a systematic review and meta-analysis, combining cognitive behavioural therapy with gradual tapering of the drugs over three months was more effective for stopping the drugs than gradual tapering alone. The combination was associated with shortterm improvement in the symptoms of insomnia. ${ }^{31}$

\section{Referral}

Referral to a sleep specialist for further evaluation should be considered in patients with insomnia who have a possible comorbid sleep disorder, do not respond to brief behavioural therapy or have persistent and distressing symptoms. The Australasian Sleep Association Sleep Services Directory is a useful resource to assist with referrals. ${ }^{32}$ When it is suspected that insomnia is related to other comorbidities such as depression or anxiety, referral to a psychiatrist should be considered.

\section{Conclusion}

Chronic insomnia disorder is a common condition that presents many challenges in its evaluation and management in primary care due to limited resources and support. Brief behavioural therapy for insomnia is an efficient management strategy that does not require specialised training and can be used effectively as first-line therapy in general practice.

Drugs may be useful as a short-term approach during episodes of acute insomnia, as an adjuvant to behavioural therapy or when there is a high level of distress. The nature of the patient's insomnia and the intended effect should be taken into consideration when prescribing. There should be a harm-benefit assessment before starting drug therapy and a plan for stopping it. Referral to specialised sleep services for complex patients is recommended. $\varangle$

\section{Conflicts of interest: none declared}

\section{REFERENCES}

1. American Psychiatric Assocation. Diagnostic and statistical manual of mental disorders. 5th edition. Arlington (VA): American Pscyhiatric Assocation; 2013.

2. Reynolds AC, Appleton SL, Gill TK, Adams RJ. Chronic insomnia disorder in Australia - a report to the Sleep Health Foundation. Sydney: Sleep Health Foundation; 2019. https://www.sleephealthfoundation.org.au/news/specialreports/chronic-insomnia-disorder-in-australia.html [cited $2021 \mathrm{Jul} 1]$

3. Buysse DJ, Angst J, Gamma A, Ajdacic V, Eich D, Rössler W. Prevalence, course, and comorbidity of insomnia and depression in young adults. Sleep 2008;31:473-80. https://doi.org/10.1093/sleep/31.4.473
4. Bertisch SM, Pollock BD, Mittleman MA, Buysse DJ, Bazzano LA, Gottlieb DJ, et al. Insomnia with objective short sleep duration and risk of incident cardiovascular disease and all-cause mortality: Sleep Heart Health Study. Sleep (Basel) 2018;41:zsy047. https://doi.org/10.1093/sleep/zsy047

5. Vgontzas AN, Liao D, Pejovic S, Calhoun S, Karataraki M, Basta $M$, et al. Insomnia with short sleep duration and mortality: the Penn State cohort. Sleep 2010;33:1159-64. https://doi.org/10.1093/sleep/33.9.1159

6. Bin YS, Marshall NS, Glozier N. The burden of insomnia on individual function and healthcare consumption in Australia. Aust N Z J Public Health 2012;36:462-8. https://doi.org/ 10.1111/j.1753-6405.2012.00845.x 
7. Daley M, Morin CM, LeBlanc M, Grégoire JP, Savard J, Baillargeon L. Insomnia and its relationship to health-care utilization, work absenteeism, productivity and accidents. Sleep Med 2009;10:427-38. https://doi.org/10.1016/ j.sleep.2008.04.005

8. Hillman D, Mitchell S, Streatfeild J, Burns C, Bruck D, Pezzullo $\mathrm{L}$. The economic cost of inadequate sleep. Sleep (Basel) 2018:41:zsy083. https://doi.org/10.1093/ sleep/zsy083

9. Cunnington $D$, Junge M. Chronic insomnia: diagnosis and non-pharmacological management. BMJ 2016;355:i5819. https://doi.org/10.1136/bmj.i5819

10. Klink ME, Quan SF, Kaltenborn WT, Lebowitz MD. Risk factors associated with complaints of insomnia in a general adult population: influence of previous complaints of insomnia. Arch Intern Med 1992;152:1634-7. https://doi.org/ 10.1001/archinte.1992.00400200070012

11. Morin CM, Bélanger L, LeBlanc M, Ivers H, Savard J, Espie CA, et al. The natural history of insomnia: a population-based 3-year longitudinal study. Arch Intern Med 2009;169:447-53. https://doi.org/10.1001/ archinternmed.2008.610

12. Roth T. Comorbid insomnia: current directions and future challenges. Am J Manag Care 2009;15 Suppl:S6-13.

13. Krakow B, Melendrez D, Ferreira E, Clark J, Warner TD, Sisley $B$, et al. Prevalence of insomnia symptoms in patients with sleep-disordered breathing. Chest 2001;120:1923-9. https://doi.org/10.1378/chest.120.6.1923

14. Bower P, Gilbody S. Stepped care in psychological therapies: access, effectiveness and efficiency. Narrative literature review. Br J Psychiatry 2005;186:11-7. https://doi.org/10.1192/ bjp.186.1.11

15. Espie CA. "Stepped care": a health technology solution for delivering cognitive behavioral therapy as a first line insomnia treatment. Sleep 2009;32:1549-58. https://doi.org/ 10.1093/sleep/32.12.1549

16. Ree M, Junge M, Cunnington D. Australasian Sleep Association position statement regarding the use of psychological/behavioral treatments in the management of insomnia in adults. Sleep Med 2017;36 Suppl 1:S43-7. https://doi.org/10.1016/j.sleep.2017.03.017

17. Morgenthaler T, Kramer M, Alessi C, Friedman L, Boehlecke B, Brown T, et al.; American Academy of Sleep Medicine. Practice parameters for the psychological and behavioral treatment of insomnia: an update. An american academy of sleep medicine report. Sleep 2006;29:1415-9. https://doi.org/10.1093/sleep/29.11.1415

18. Fernando A 3rd, Arroll B, Falloon K. A double-blind randomised controlled study of a brief intervention of bedtime restriction for adult patients with primary insomnia. J Prim Health Care 2013·5·5-10. https://doi.org/10.1071/HC13005

19. Buysse DJ, Germain A, Moul DE, Franzen PL, Brar LK, Fletcher ME, et al. Efficacy of brief behavioral treatment for chronic insomnia in older adults. Arch Intern Med 2011;171:887-95. https://doi.org/10.1001/ archinternmed.2010.535

20. Royal Australian College of General Practitioners. HAND Interventions: Brief behavioural therapy: insomnia in adults. Melbourne: RACGP; 2014. https://www.racgp.org.au/clinicalresources/clinical-guidelines/handi/handi-interventions/ mental-health/brief-behavioural-therapy-insomnia-in-adults [cited $2021 \mathrm{Jul} 1]$
21. Trauer JM, Qian MY, Doyle JS, Rajaratnam SM, Cunnington D. Cognitive behavioral therapy for chronic insomnia: a systematic review and meta-analysis. Ann Intern Med 2015;163:191-204. https://doi.org/10.7326/M14-2841

22. Morin CM, Vallières A, Guay B, Ivers $H$, Savard J, Mérette $C$, et al. Cognitive behavioral therapy, singly and combined with medication, for persistent insomnia: a randomized controlled trial. JAMA 2009:301:2005-15. https://doi.org/10.1001/ jama.2009.682

23. Sateia MJ, Buysse DJ, Krystal AD, Neubauer DN, Heald JL. Clinical practice guidelines for pharmacological treatment of chronic insomnia in adults: an American Academy of Sleep Medicine clinical practice guideline. J Clin Sleep Med 2017;13:307-49. https://doi.org/10.5664/jcsm.6470

24. Lemoine P, Nir T, Laudon M, Zisapel N. Prolonged-release melatonin improves sleep quality and morning alertness in insomnia patients aged 55 years and older and has no withdrawal effects. J Sleep Res 2007;16:372-80. https://doi.org/10.1111/j.1365-2869.2007.00613 x

25. Lack LC, Wright HR. Treating chronobiological components of chronic insomnia. Sleep Med 2007;8:637-44. https://doi.org/10.1016/j.sleep.2006.10.003

26. Herring WJ, Connor KM, Snyder E, Snavely DB, Zhang Y, Hutzelmann J, et al. Suvorexant in patients with insomnia: pooled analyses of three-month data from phase-3 randomized controlled clinical trials. J Clin Sleep Med 2016;12:1215-25. https://doi.org/10.5664/jcsm.6116

27. Nowell PD, Mazumdar S, Buysse DJ, Dew MA, Reynolds CF 3rd, Kupfer DJ. Benzodiazepines and zolpidem for chronic insomnia: a meta-analysis of treatment efficacy. JAMA 1997;278:2170-7. https://doi.org/10.1001/ jama.1997.03550240060035

28. Wiegand $\mathrm{MH}$. Antidepressants for the treatment of insomnia : a suitable approach? Drugs 2008:68:2411-7. https://doi.org/10.2165/0003495-200868170-00001

29. Thompson W, Quay TA, Rojas-Fernandez C, Farrell B, Bjerre LM. Atypical antipsychotics for insomnia: a systematic review. Sleep Med 2016;22:13-7. https://doi.org/10.1016/ .sleep.2016.04.003

30. Vande Griend JP, Anderson SL. Histamine-1 receptor antagonism for treatment of insomnia. J Am Pharm Assoc (2003) 2012;52:e210-9. https://doi.org/10.1331/ JAPhA.2012.12051

31. Takaesu Y, Utsumi T, Okajima I, Shimura A, Kotorii N, Kuriyama $\mathrm{K}$, et al. Psychosocial intervention for discontinuing benzodiazepine hypnotics in patients with chronic insomnia: A systematic review and meta-analysis. Sleep Med Rev 2019;48:101214. https://doi.org/10.1016/j.smrv.2019.101214

32. Australasian Sleep Association. Sleep Services Directory. https://sleep.org.au/Public/Resources/SleepDirectory/Public/Resource-Centre/Sleep-services. aspx?hkey=046a0d7d-338b-46c7-a94c-f65cbc950f17 [cited $2021 \mathrm{Jul} 1]$ 